Supporting Information of

\title{
Competing Supramolecular Assembly of Amphiphiles to Form Micelles or Pseudorotaxanes
}

\author{
Yuji Suzaki, Toshiaki Taira, Daisuke Takeuchi and Kohtaro Osakada* \\ Chemical Resources Laboratory R1-3, Tokyo Institute of Technology \\ 4259 Nagatsuta, Midori-ku, Yokohama 226-8503, Japan. \\ Fax: +81-45-924-5224; Tel: +81-45-924-5224 \\ E-mail: kosakada@res.titech.ac.jp
}

\section{Experimental Section}

General. The hydrodynamic size of micelles in an aqueous solution was measured using an Otsuka Electronics Co., Ltd. DLS-7000 equipped with a $10 \mathrm{~mW}$ He-Ne laser operating at $632.8 \mathrm{~nm}$. Measurement was performed at an angle of $90^{\circ}$, and the data obtained were fitted using the CONTIN algorithm. The absorption spectrum of pyrene was recorded using a JASCO V-530 UV/vis spectrometer. A $10-\mu \mathrm{L}$ aliquot of a $2 \mathrm{mM}$ solution of pyrene in dimethyl sulfoxide (DMSO) was transferred to $2 \mathrm{~mL}$ of each sample. Before measuring, the samples were stored at an adequate temperature using a JASCO EHC-477 peltire-type thermostated cell holder. Synthesis of 1 was described in elsewhere. ${ }^{1}$

$\mathbf{C l}\left(\mathrm{CH}_{2}\right)_{10} \mathrm{OC}_{6} \mathbf{H}_{3}-3,5-(\mathrm{OMe})_{2}$ : A solution of 3,5-dimethoxyphenol (4.0 g, $\left.26 \mathrm{mmol}\right)$ and $\mathrm{NaOH}$ in DMF was stirred for $1 \mathrm{~h}$ at room temperature, followed by the addition of 1,10-dichlorodecane $(11 \mathrm{~mL}, 52 \mathrm{mmol})$. The mixture was stirred for an other $22 \mathrm{~h}$ at 100 ${ }^{\circ} \mathrm{C}$. The water layer was extracted with $\mathrm{Et}_{2} \mathrm{O}(30 \mathrm{~mL}, 3$ times $)$ and the combined organic extract was washed with $1 \mathrm{M} \mathrm{HCl}$ in water $\left(30 \mathrm{~mL}, 3\right.$ times), dried over $\mathrm{MgSO}_{4}$, filtered and concentrated under reduced pressure to yield a crude product, which was purified by $\mathrm{SiO}_{2}$ column chromatography (hexane/ $\mathrm{CH}_{2} \mathrm{Cl}_{2}=5 / 1$ ). Further purification of the product by recrystallization at $20{ }^{\circ} \mathrm{C}$ and washing with cold hexane yields $\mathrm{Cl}\left(\mathrm{CH}_{2}\right)_{10} \mathrm{OC}_{6} \mathrm{H}_{3}-3,5-(\mathrm{OMe})_{2}$ as a white powder $(2.14 \mathrm{~g}, 6.5 \mathrm{mmol}, 25 \%) .{ }^{1} \mathrm{H} \mathrm{NMR}$ 
(300 MHz, $\mathrm{CDCl}_{3}$, r.t.): $\delta=1.31-1.42\left(12 \mathrm{H} ; \mathrm{CH}_{2}\right), 1.74-1.79\left(4 \mathrm{H} ; \mathrm{CH}_{2}\right), 3.53\left(\mathrm{t},{ }^{3} J(\mathrm{H}\right.$, $\left.\mathrm{H})=7 \mathrm{~Hz}, 2 \mathrm{H} ; \mathrm{CH}_{2}\right), 3.77\left(\mathrm{~s}, 6 \mathrm{H} ; \mathrm{OCH}_{3}\right), 3.91\left(\mathrm{t},{ }^{3} \mathrm{~J}(\mathrm{H}, \mathrm{H})=7 \mathrm{~Hz}, 2 \mathrm{H} ; \mathrm{CH}_{2}\right), 6.07-6.09$ (3H; ortho- $\mathrm{C}_{6} \mathrm{H}_{3}$, para- $\left.\mathrm{C}_{6} \mathrm{H}_{3}\right) ;{ }^{13} \mathrm{C}\left\{{ }^{1} \mathrm{H}\right\} \mathrm{NMR}\left(75.5 \mathrm{MHz}, \mathrm{CDCl}_{3}\right.$, r.t.): $\delta=26.0\left(\mathrm{CH}_{2}\right), 26.8$ $\left(\mathrm{CH}_{2}\right), 28.8\left(\mathrm{CH}_{2}\right), 29.2\left(\mathrm{CH}_{2}\right), 29.3\left(\mathrm{CH}_{2}\right), 29.4\left(\mathrm{CH}_{2}\right), 32.6\left(\mathrm{CH}_{2}\right), 45.2\left(\mathrm{ClCH}_{2}\right), 55.3$ $\left(\mathrm{OCH}_{3}\right), 67.9\left(\mathrm{OCH}_{3}\right), 92.7$ (ortho- $\left.\mathrm{C}_{6} \mathrm{H}_{3}\right), 93.3\left(\right.$ para- $\left.\mathrm{C}_{6} \mathrm{H}_{3}\right), 161.0\left(\right.$ meta- $\left.\mathrm{C}_{6} \mathrm{H}_{3}\right), 161.4$ (ipso- $\mathrm{C}_{6} \mathrm{H}_{3}$ ); Anal. Calcd. for $\mathrm{C}_{18} \mathrm{H}_{29} \mathrm{ClO}_{3}$ : C, 65.74; $\mathrm{H}, 8.89 ; \mathrm{N}, \mathrm{Cl}, 10.78$. Found: $\mathrm{C}$, 65.76; H, 8.86; Cl, 10.61 .

[4,4'-bpy- $\left.\mathrm{N}-\left(\mathrm{CH}_{2}\right)_{10} \mathrm{OC}_{6} \mathrm{H}_{3}-3,5-(\mathrm{OMe})_{2}\right]^{+}\left(\mathrm{Cl}^{-}\right) \quad$ (2): $\quad$ A solution of $\mathrm{Cl}\left(\mathrm{CH}_{2}\right)_{10} \mathrm{OC}_{6} \mathrm{H}_{3}-3,5-(\mathrm{OMe})_{2}(1.0 \mathrm{~g}, 3 \mathrm{mmol})$ and 4,4'-bipyridine (945 mg, $\left.6 \mathrm{mmol}\right)$ in DMF was stirred at $100{ }^{\circ} \mathrm{C}$ for $24 \mathrm{~h}$. The evaporation of the solvent yielded a brown solid, which was purified by washing with $\mathrm{Et}_{2} \mathrm{O}(50 \mathrm{~mL}, 3$ times $)$, recrystallization from $\mathrm{CH}_{2} \mathrm{Cl}_{2} / \mathrm{Et}_{2} \mathrm{O}(7 \mathrm{~mL} / 50 \mathrm{~mL})$ at room temperature, and washing with $\mathrm{Et}_{2} \mathrm{O}$ to yield 2 , a pale brown solid (405 mg, $0.8 \mathrm{mmol}, 27 \%) .{ }^{1} \mathrm{H} \mathrm{NMR}\left(300 \mathrm{MHz}, \mathrm{CDCl}_{3}\right.$, r.t.): $\delta=1.27-1.37\left(12 \mathrm{H} ; \mathrm{CH}_{2}\right), 1.73\left(\mathrm{~m}, 2 \mathrm{H} ; \mathrm{CH}_{2}\right), 2.06\left(\mathrm{~m}, 2 \mathrm{H} ; \mathrm{CH}_{2}\right), 3.75\left(\mathrm{~s}, 6 \mathrm{H} ; \mathrm{OCH}_{3}\right), 3.88$

$\left(\mathrm{t},{ }^{3} J(\mathrm{H}, \mathrm{H})=7 \mathrm{~Hz}, 2 \mathrm{H} ; \mathrm{CH}_{2}\right), 5.04\left(\mathrm{t},{ }^{3} \mathrm{~J}(\mathrm{H}, \mathrm{H})=7 \mathrm{~Hz}, 2 \mathrm{H} ; \mathrm{NCH}_{2}\right), 6.05-6.07(3 \mathrm{H}$; ortho- $\mathrm{C}_{6} \mathrm{H}_{3}$, para- $\left.\mathrm{C}_{6} \mathrm{H}_{3}\right), 7.85\left(\mathrm{~d},{ }^{3} J(\mathrm{H}, \mathrm{H})=5 \mathrm{~Hz}, 2 \mathrm{H} ; \mathrm{C}_{10} \mathrm{H}_{8} \mathrm{~N}_{2}\right), 8.44\left(\mathrm{~d},{ }^{3} J(\mathrm{H}, \mathrm{H})=6 \mathrm{~Hz}\right.$, $\left.2 \mathrm{H} ; \mathrm{C}_{10} \mathrm{H}_{8} \mathrm{~N}_{2}\right), 8.89\left(\mathrm{~d},{ }^{3} J(\mathrm{H}, \mathrm{H})=6 \mathrm{~Hz}, 2 \mathrm{H} ; \mathrm{C}_{10} \mathrm{H}_{8} \mathrm{~N}_{2}\right), 9.57\left(\mathrm{~d},{ }^{3} J(\mathrm{H}, \mathrm{H})=6 \mathrm{~Hz}, 2 \mathrm{H}\right.$; $\left.\mathrm{C}_{10} \mathrm{H}_{8} \mathrm{~N}_{2}\right) ;{ }^{13} \mathrm{C}\left\{{ }^{1} \mathrm{H}\right\} \mathrm{NMR}\left(75.5 \mathrm{MHz}, \mathrm{CDCl}_{3}\right.$, r.t.): $\delta=25.8\left(\mathrm{CH}_{2}\right), 26.0\left(\mathrm{CH}_{2}\right), 28.9\left(\mathrm{CH}_{2}\right)$, $29.0\left(\mathrm{CH}_{2}\right), 29.1\left(\mathrm{CH}_{2}\right), 29.2\left(\mathrm{CH}_{2}\right), 31.7\left(\mathrm{CH}_{2}\right), 45.2,55.2\left(\mathrm{OCH}_{3}\right), 61.6\left(\mathrm{NCH}_{2}\right), 67.8$ $\left(\mathrm{OCH}_{2}\right), 92.6$ (ortho- $\left.\mathrm{C}_{6} \mathrm{H}_{3}\right), 93.2\left(\right.$ para- $\left.\mathrm{C}_{6} \mathrm{H}_{3}\right), 121.6\left(\mathrm{C}_{10} \mathrm{H}_{8} \mathrm{~N}_{2}\right), 125.8\left(\mathrm{C}_{10} \mathrm{H}_{8} \mathrm{~N}_{2}\right), 141.0$ $\left(\mathrm{C}_{10} \mathrm{H}_{8} \mathrm{~N}_{2}\right), 125.7\left(\mathrm{C}_{10} \mathrm{H}_{8} \mathrm{~N}_{2}\right), 151.0\left(\mathrm{C}_{10} \mathrm{H}_{8} \mathrm{~N}_{2}\right), 153.2\left(\mathrm{C}_{10} \mathrm{H}_{8} \mathrm{~N}_{2}\right), 160.8$ (meta- $\left.\mathrm{C}_{6} \mathrm{H}_{3}\right)$, 161.3 (ipso- $\left.\mathrm{C}_{6} \mathrm{H}_{3}\right)$; Anal. Calcd. for $\mathrm{C}_{28} \mathrm{H}_{37} \mathrm{ClN}_{2} \mathrm{O}_{3}+1.5\left(\mathrm{H}_{2} \mathrm{O}\right)$ : C, 65.67; H, 7.87; N, 5.47; Cl, 6.92. Found: C, 65.99; H, 8.14; N, 5.74; Cl, 7.06.

Reference

[1] Y. Suzaki, T. Taira, K. Osakada, Dalton Trans. 2006, 5345. 


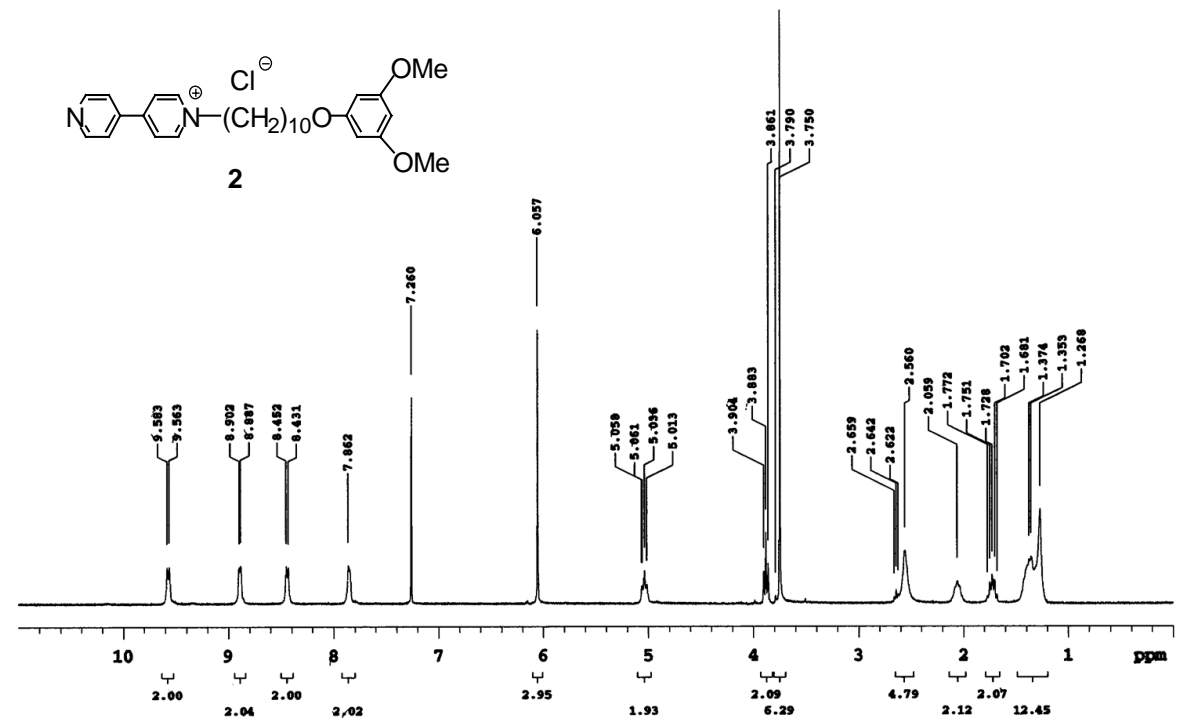

Figure S1. ${ }^{1} \mathrm{H}$ NMR spectrum of 2. $\left(300 \mathrm{MHz}, \mathrm{CDCl}_{3}\right.$, r.t.)

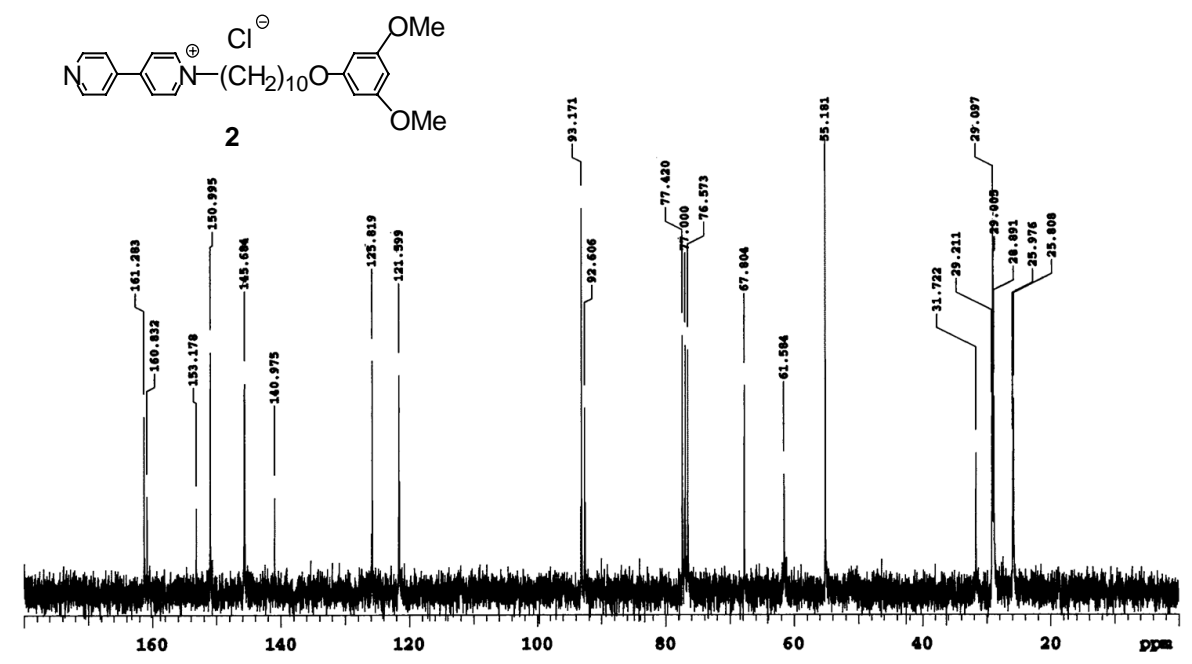

Figure S2. ${ }^{13} \mathrm{C}\left\{{ }^{1} \mathrm{H}\right\}$ NMR spectrum of 2. $\quad\left(75.5 \mathrm{MHz}, \mathrm{CDCl}_{3}\right.$, r.t.) 


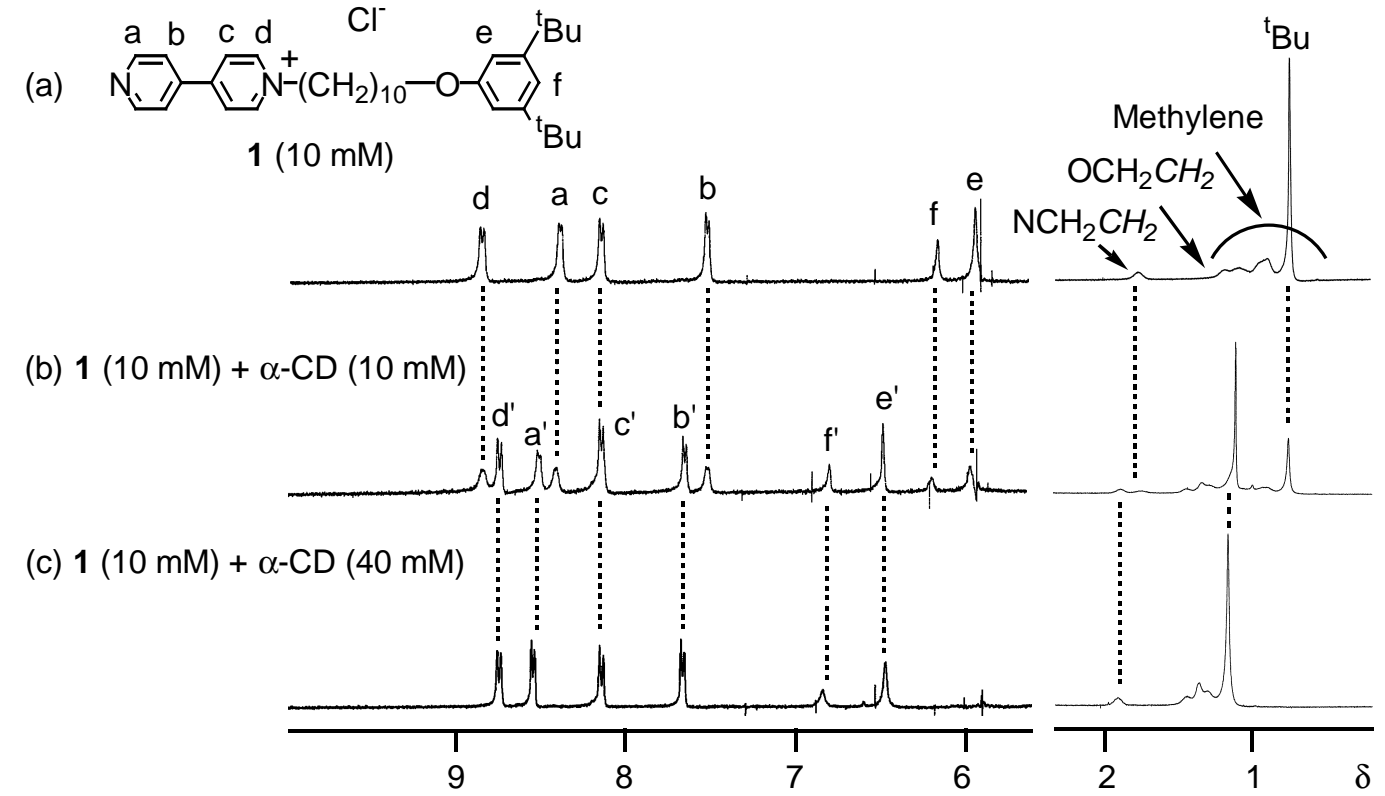

Figure S3. ${ }^{1} \mathrm{H}$ NMR spectra of (a) $1(10 \mathrm{mM})$, (b) $\mathbf{1}(10 \mathrm{mM})+\alpha-\mathrm{CD}(10 \mathrm{mM})$, and (c) $1(10 \mathrm{mM})+\alpha-\mathrm{CD}(40 \mathrm{mM}) . \quad\left(300 \mathrm{MHz}, \mathrm{D}_{2} \mathrm{O}\right.$, r.t. $)$

(a)

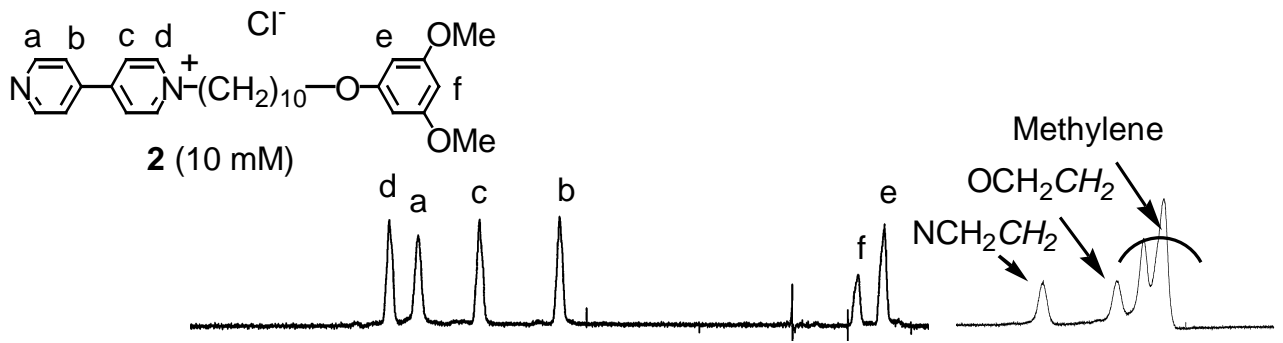

(b) $2(10 \mathrm{mM})+\alpha-C D(10 \mathrm{mM})$

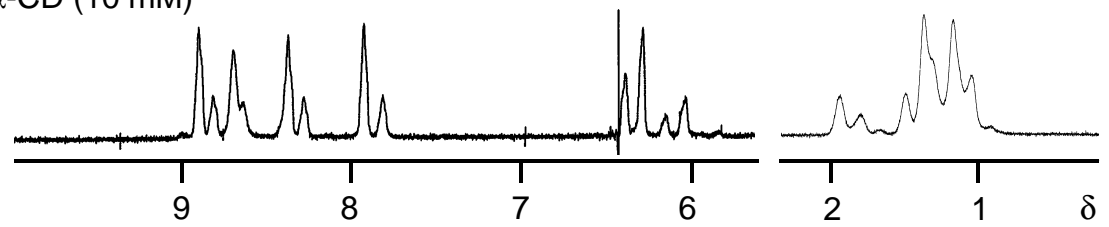

Figure S4. $\quad{ }^{1} \mathrm{H}$ NMR spectra of (a) $2(10 \mathrm{mM})$, (b) $2(10 \mathrm{mM})+\alpha-\mathrm{CD}(10 \mathrm{mM}) . \quad$ (300 $\mathrm{MHz}, \mathrm{D}_{2} \mathrm{O}$, r.t.) 